\title{
Dinámica del fósforo en un agroecosistema conservacionista maíz-ganado de sabanas neotropicales
}

\section{Phosphorus dynamics in conservationist agroecosystem maize-cattle of neotropical savanna}

\author{
Ramírez-Iglesias Elizabeth ${ }^{1 *}$ Ph.D; Hernández-Hernández Rosa Mary ${ }^{1}$ Ph.D; Herrera Pablo M.Sc.
}

${ }^{1}$ Universidad Nacional Experimental Simón Rodríguez. Instituto de Estudios Científicos y Tecnológicos (UNESR-IDECYT). Caracas, Venezuela.

Keywords:

Forage;

relationship soil-plant-excreta;

cover;

vegetal nutrition.

Palabras Clave:

Forraje; relación suelo-planta-excretas; coberturas: nutrición vegetal.

\section{Abstract}

Phosphorus is one of the limiting elements in acid soils, so the incorporation of strategies for its cycling within an agroecosystem is one of the key variables, in order to optimize agricultural production. The dynamics of phosphorus in a maize-livestock agroecosystem were evaluated under the types of forages introduced: Brachiaria dyctioneura $(\mathrm{Bd})$, a grass and Centrosema macrocarpum $(\mathrm{Cm})$, a legume, which in turn served as associated perennial coverages maize grown with direct sowing. The dynamics of this element are contrasted in the savanna of natural vegetation (SN). It determined the availability of this element in the soil $(0-15 \mathrm{~cm})$, as well as the total $P$ content in the excreta of the cattle and in the green fraction of the vegetation during the dry season, after the harvest of the corn, when the cattle a grazing is introduced. We analyzed the bromatological quality of livestock and the daily gain of weight in livestock (GDP). Concentrations of total $\mathrm{P}$ in the plant biomass varied according to the quality of the pasture forage, being higher in the $\mathrm{Cm}$. This significantly affected $(p<0.01)$ the excreta $\mathrm{P}$ and the available $\mathrm{P}$ of the soil, which resulted in a higher GDP. In the savanna conditions, $\mathrm{P}$ cycling in the subsystems of soil-plant-animal, is more effective when a forage legume is used as $\mathrm{Cm}$, since it favors the incorporation of $\mathrm{P}$ through the excreta and by the decomposition of his necromass.

\section{Resumen}

El fósforo constituye uno de los elementos limitantes en suelos ácidos, por lo que la incorporación de estrategias para su ciclaje dentro de un agroecosistema es una de las variables claves, a fin de optimizar la producción agropecuaria. Se evaluó, la dinámica del fósforo en un agroecosistema conservacionista maíz-ganado, bajo dos tipos de forrajes introducidos: Brachiaria dyctioneura (Bd), una gramínea y Centrosema macrocarpum $(\mathrm{Cm})$, una leguminosa, que a su vez se desempeñaron como coberturas perennes asociadas al maíz cultivado con siembra directa. Se contrastó la dinámica de este elemento en la sabana de vegetación natural (SN). Se determinó la disponibilidad de este elemento en el suelo $(0-15 \mathrm{~cm})$, así como el $P$ total contenido en las heces del ganado y en la fracción verde de la vegetación durante la época seca, después de la cosecha del maíz, cuando el ganado se introduce a pastoreo. Se analizó la calidad bromatológica del forraje y la ganancia diaria de peso en el ganado (GDP). Las concentraciones de $P$ total en la biomasa vegetal varió de acuerdo a la calidad del forraje pastoreado, siendo mayor en la $\mathrm{Cm}$. Ello incidió significativamente $(p<0.01)$ en el $\mathrm{P}$ de las excretas y en el $\mathrm{P}$ disponible del suelo, lo cual resultó en una mayor GDP. En condiciones de sabana, el ciclaje del $P$ en los subsistemas suelo-planta-animal, es más efectivo cuando se usa una leguminosa forrajera como $\mathrm{Cm}$, ya que favorece la incorporación del $\mathrm{P}$ a través de las excretas y por la descomposición de su necromasa. 


\section{Introducción}

En los suelos de las sabanas neotropicales, el fósforo $(P)$ es uno de los elementos más deficientes después del nitrógeno $(\mathrm{N})$, a pesar de ser relativamente estable en el suelo, ya que no presenta compuestos inorgánicos volátiles o fácilmente lixiviables (PORTELA et al., 2016). Este elemento no solo limita la fertilidad de los suelos, sino que también afecta la calidad química de las pasturas e incide en la respuesta del ganado durante el pastoreo (SEMMARTIN et al., 2010).

En Venezuela, HERNÁNDEZ-HERNÁNDEZ et al (2000) han planteado una alternativa de transición agroecológica, a través de la cual se trabaja en la transformación de sistemas convencionales de producción mixta, basados en monocultivos de maíz y ganadería extensiva, hacia sistemas conservacionistas; introduciendo la siembra directa y la asociación de coberturas perennes con maíz, a fin de diversificar el agroecosistema, darle protección al suelo; conservando su humedad (SANABRÍA et al., 2006), aumentando la actividad biológica (PORTELA et al., 2016) y facilitando el ciclaje de nutrientes por la descomposición de una necromasa de mejor calidad nutricional (THOMAS Y ASAKAWA, 1993) y ofreciendo al ganado bovino y ovino una mejor oferta forrajera en una época crítica para el ganado, como lo es la época seca (RAMÍREZIGLESIAS, 2015).

Para lograr una comprensión integral del agroecosistema considerando las relaciones entre los subsistemas sueloplanta-animal, tomando como eje central, las dinámicas del $\mathrm{P}$ en cada uno de sus componentes, se explora el comportamiento de éste elemento en el suelo, en la hoja verde de dos tipos de forraje Brachiaria dyctioneura (Bd) una gramínea, y Centrosema macrocarpum $(\mathrm{Cm})$ una leguminosa, contrastando con la sabana de vegetación natural (SN). Asimismo, y de forma simultánea se evaluó el $\mathrm{P}$ presente en las excretas del ganado que pastoreó este agroecosistema, así como las posibles correlaciones entre el $\mathrm{P}$ disponible del suelo, el $\mathrm{P}$ a nivel foliar y a su vez las posibles entradas de este elemento mediante las excretas, a fin de tener una visión general del ciclaje del $\mathrm{P}$ en los compartimentos mencionados del agroecosistema evaluado.

\section{Materiales y métodos}

Características de la zona de estudio: La investigación se desarrolló en la Estación Experimental "La Iguana", perteneciente a la Universidad Nacional Experimental "Simón Rodríguez", ubicada geográficamente a $8^{\circ}$ $25^{\prime} \mathrm{N}$ y $65^{\circ} 25^{\prime} \mathrm{O}$ y una altitud entre $80-120 \mathrm{msnm}$ (RIERA y GUERRERO, 1984), en las sabanas del sur-oriente del estado Guárico, en los llanos centrales venezolanos a $80 \mathrm{~km}$ al sur-este de la población de
Santa María de Ipire. Según la clasificación de EWEL et al. (1976), la estación se ubica en la zona de vida de bosque seco tropical. El clima es estacional con dos periodos contrastantes muy marcados; uno seco desde noviembre a mayo y otro lluvioso de junio a octubre. La precipitación total anual oscila entre 950 y 1300 $\mathrm{mm}$, y las temperaturas medias mensuales entre 26 y $30^{\circ} \mathrm{C}$, siendo los meses más calurosos marzo, abril y mayo (MATA et al.,1996). Predominan las sabanas bien drenadas o sabanas de Trachypogon (RAMIA, 1967), caracterizadas por grandes extensiones de gramíneas, dominadas por los géneros de Trachypogon y Axonopus, con árboles dispersos de las especies Curatella americana, Byrsonima crassifolia y Bowdichia virgilioides. Fisiográficamente puede caracterizarse como una altiplanicie de mesa conservada combinada con depósitos eólicos y algunos afloramientos del material terciario, la pendiente general es de $1-3 \%$, con un predominio de un suelo de tipo Ultisol de textura arenosa, con un drenaje interno rápido a moderadamente rápido (LOZANO et al., 2010). En general los suelos poseen fertilidad de baja a muy baja, con un pH promedio de 5,2 pero puede variar entre 4,5 y 5,8 (HERNÁNDEZ-HERNÁNDEZ et al., 2000). Los análisis de suelo con fines de fertilidad reportan valores de $\mathrm{P}$ muy bajos y su valor promedio es de 2 $\mathrm{mg} \cdot \mathrm{kg}^{-1}$; el K también es bajo con valores de $50 \mathrm{mg} \cdot \mathrm{kg}^{-1}$ aproximadamente, y el Ca con valores de $120 \mathrm{mg}^{\mathrm{kg}}{ }^{-1}$ y $1,7 \%$ de materia orgánica $(\mathrm{MO})$ también considerado como bajo (LOZANO et al., 2010).

Historia de manejo: En el marco del proyecto: "Manejo Agroecológico de suelos de sabanas bien drenadas, con unidades de producción cereal-ganado" se estableció un agroecosistema de maíz-ganado en suelos de sabanas bien drenadas, usando la siembra directa, asociación del maíz con coberturas de gramíneas y leguminosas y diferentes tipos de fertilización fosforada. Para el año 2002 se introdujeron dos tipos de coberturas perennes en el agroecosistema: la leguminosa Centrosema macrocarpum y la gramínea Brachiaria dyctioneura, considerándose la vegetación natural del ecosistema de la sabana como el tratamiento testigo, en parcelas adyacentes al área experimental. Ambas coberturas se establecieron durante dos años. Al cabo de ese tiempo, se realizó un primer corte de las coberturas para la siembra directa del cultivo de maíz.

El diseño experimental contempló los tratamientos de coberturas, como lo fue la Brachiaria dyctioneura, Centrosema macrocarpum y el control, vegetación natural de sabana. En los suelos de todos los ensayos y para el establecimiento de las coberturas en el año 2002 se añadió una fertilización básica de 150 - 150 - $100 \mathrm{~kg} \mathrm{ha}^{-1} \mathrm{~N}-\mathrm{P}_{2} \mathrm{O}_{5}-\mathrm{K}_{2} \mathrm{O}$, utilizándose Urea y Sulfato doble de potasio y magnesio. 
Dentro de lo que sería el manejo conservacionista de la fertilización en suelos arenosos, se fraccionó la dosis de fertilizante en tres partes; a la siembra se aplicó todo el $\mathrm{P}_{2} \mathrm{O}_{5}$, así como un tercio de la dosis de $\mathrm{N}$, y la mitad de la dosis de K. A los 20 días después de la siembra (dds) se repitió las dosis de $\mathrm{N}$ y K y a los 40 dds sólo se añadió un tercio de $\mathrm{N}$.

La aplicación de fertilizantes fue realizada en "hilo", al momento de la siembra del cultivo, puesto que se usó una sembradora CSEMEATO de siembra directa que abona y siembra al mismo tiempo. Se utilizó semilla de maíz certificada -HIMECA 3005-, aplicada con una densidad de siembra de 60.000 plantas. ha $^{-1}$ (LOZANO et al., 2010). Se usó un herbicida de contacto -paraquaten una dosis baja, a razón de 2 L.ha-1 a fin de atenuar el crecimiento de las coberturas y dar ventaja al crecimiento del maíz.

Previo al momento de la siembra del maíz, las coberturas establecidas se cortaron a ras del suelo con rotativa, y los residuos se dejaron sobre la superficie. La siembra de este cereal se realizó con la sembradora de siembra directa sobre la necromasa de las coberturas, las cuales rebrotaron estimuladas por la fertilización que se realizó previamente. El crecimiento de estos rebrotes fue inferior al del cultivo, lo que impidió un efecto negativo sobre los primeros estadios del maíz. Una vez realizada la cosecha del cereal, al inicio de la época seca, se introdujo el ganado, para que se alimentaran de las coberturas y del rastrojo de maíz, por un período de 110 días.

Diseño experimental: El diseño experimental del ensayo correspondió al de parcelas grandes sin repetición, para el cual se realizó un estudio previo de variabilidad espacial (MACHADO, 2000), de manera de obtener un diseño que permitiera utilizar áreas de suelos similares heterogéneas. Con ello se estableció la orientación y el tamaño de las parcelas, al igual que el número de muestras representativas para el suelo (HERNÁNDEZ-HERNÁNDEZ et al., 2000).

Así los tratamientos de coberturas se establecieron en parcelas de $400 \times 72 \mathrm{~m}$. Con fines de orientación en el sitio, se definieron sub-parcelas de $60 \times 15 \mathrm{~m}$ separadas por espacios de $40 \mathrm{~cm}$ para los muestreos de suelo y vegetación. Por otra parte, cada parcela de tratamiento de cobertura se dividió en dos sectores (potreros), a fin de ubicar a los animales y así obtener repeticiones por cada tratamiento de cobertura. Se establecieron 4 potreros, dividiendo las parcelas de cada cobertura en dos sectores, de manera de tener repeticiones de animales por tratamiento, tanto para la $\mathrm{Bd}$, como para la $\mathrm{Cm}$. Las dimensiones de los potreros fueron de $200 \mathrm{x}$ $72 \mathrm{~m}$, con una carga animal estimada de 1,04 UA.ha-1. Se trabajó con 36 becerros destetados en total, del tipo mestizo criollo limonero con predominancia de sangre cebú, 16 por cada cobertura introducida, y en el caso de la SN se trabajó con 4 animales. Todo los becerros se vacunaron y desparasitaron 2 semanas antes de introducirlos en el ensayo. Previamente se hizo un periodo de adaptación a los potreros, con la finalidad de acostumbrar a los bovinos al forraje ofrecido y a las barreras utilizadas como cerca eléctrica (RODASGONZALEZ et al., 2006).

Para evaluar de forma integral el efecto de las coberturas (gramínea y leguminosa) sobre la dinámica del P en los componentes suelo-planta y su posible contribución de este elemento al suelo, mediante las excretas, se realizó su seguimiento de manera simultánea en el suelo, las coberturas y las excretas del rebaño, al inicio del pastoreo, luego a los 60 días y finalmente a los 110 días de introducidos los animales a pastoreo.

\section{Muestreo}

Coberturas: en el agroecosistema maíz-ganado y el ecosistema de sabana de vegetación natural, se cuantificó la biomasa total aérea mediante el uso de un aro de $50 \mathrm{~cm}$ de diámetro, cortando todo el material vegetal sobre la superficie del suelo que quedó encerrado en el aro. El mismo se lanzó al azar un total de 24 veces por cada tratamiento de cobertura en cada momento de muestreo durante el ciclo de pastoreo, posteriormente se procedió a separar por planta, las hojas verdes-ya que estas son más palatables para el ganado durante el pastoreo- y secar todo el material a $40^{\circ} \mathrm{C}$ y pesarlo a las 48 horas (STEYERMARK $Y$ HORWITZ, 1977; BROWN, 1997).

Suelo: para el subsistema suelo el muestreo fue simultáneo al de la biomasa y fue tomado a una profundidad de $0-15 \mathrm{~cm}$, similar al muestreo de biomasa se consideró tomar la muestra de suelo en el mismo lugar y de forma simultánea dónde se tomó la de vegetación.

Ganado: en el caso del componente animal, a los mautes se les tomó el peso a fin de determinar durante el ciclo la dinámica de la ganancia diaria de peso (GDP), cada 20 días durante el tiempo que duró el pastoreo, considerando siempre la misma hora del día y cercano al ensayo para evitar estrés por parte del animal. Las excretas se colectaron directamente del recto del ganado (aproximadamente $1 \mathrm{~kg}$ ), a fin de evitar su contaminación por suelo, pelos y/u otros materiales del medio. Posteriormente, se colocaron en viales de aluminio identificados con la fecha, potrero de procedencia y tipo cobertura.

Metodología analítica. Para evaluar la dinámica del P en el agroecosistema y el efecto de los distintos tipos de coberturas, se realizó la determinación de éste 
elemento de forma simultánea, tanto en la biomasa vegetal como en el suelo y en las excretas del ganado. Las muestras de heces, se secaron a $55^{\circ} \mathrm{C}$ por 48 horas, eliminando así la humedad presente, luego se molieron en un molino Wiley con malla de $1 \mathrm{~mm}$ (BALDIZÁN et al., 2006; CASTELLARO et al., 2007) y se homogeneizaron. Tanto para las muestras de excretas como para las de biomasa se realizó una digestión previa con ácido sulfúrico y su determinación colorimétrica fue por MURPHY y RILEY (1962), a fin de determinar la concentración de $P$ total. De igual manera en la biomasa se determinó los contenidos de proteína cruda, lignina, fibra detergente ácida (FDA) y fibra detergente neutra (FDN) (SOEST, 1991), para finalizar a las muestras de suelo se les determinó, el fósforo disponible empleando para ello extracción con $\mathrm{NaHCO}_{3}$, por el método Olsen (WATABLE y OLSEN, 1965) y su determinación colorimétrica fue por MURPHY y RILEY (1962).

Análisis estadístico. Se empleó un análisis descriptivo de los datos y un análisis de varianza. Asimismo, se utilizó un conjunto de unidades experimentales para valorar y comparar las respuestas obtenidas por efecto de la Brachiaria dyctioneura (Bd) y Centrosema macrocarpum $(\mathrm{Cm})$ con la vegetación nativa de la sabana natural ( $\mathrm{SN}$ ), teniendo un número de muestras para el subsistema planta de 1080, para el subsistema suelo de 540 y en el caso de las excretas del ganado fue de 108 en total.

El modelo estadístico utilizado fue:

$Y_{i j k}=\mu+C_{i}+S_{j}+G_{k}+\varepsilon_{i j k}$

Dónde:

Yijk = es la respuesta de la variable en el iésimo tratamiento (coberturas) y la k- ésimo nivel del factor $B$ (ganado).

$\mu=$ media poblacional

$\mathrm{C}_{\mathrm{i}}=$ efecto de las coberturas en el iésimo tratamiento

$S_{i}=$ representa el efecto del j-ésimo nivel del factor suelo.

$G_{k}=$ representa el efecto del k-ésimo nivel del factor ganado.

$\varepsilon_{\mathrm{ijk}}=$ error aleatorio asociado a la observación ijk-ésima

Los valores $\varepsilon_{\mathrm{ijk}}$ usualmente se suponen normales, independientes, con esperanza cero y varianza común $\delta^{2}$. En el presente diseño se consideraron repeticiones a las medidas tomadas en tres momentos de la evaluación. Bajo este modelo experimental se trabajó con correlaciones de Pearson y regresiones lineales. Como prueba para la comparación se usó la prueba de DUNCAN (1974), por presentarse para este diseño como la más potente, con un nivel de significancia de $p<0.05$ y $P<0.01$. El programa estadístico empleado fue InfoStat (DI RIENZO et al., 2011).

\section{Resultados y Discusión}

Contenido bromatológico de las coberturas durante la época de pastoreo: En la Tabla 1 se reporta que el contenido de proteína cruda (PC) en promedio y expresado con su desviación standard de $\mathrm{Cm}$ fue de $11,15 \pm 0,41 \%$, el cual está por encima del valor crítico para la alimentación en bovinos que abarca un rango de 7 a 8\% (LASCANO et al., 2002; CHACÓN, 2007), en contraste con $\mathrm{Bd}(6,70 \pm 0,45 \%)$ y la $\mathrm{SN}(5,63 \pm$ $0,77 \%$ ) cuyos promedios están por debajo de los valores críticos reportados.

Tabla 1. Contenidos de proteína cruda y lignina en las coberturas introducidas y la sabana natural durante el pastoreo en un agroecosistema maíz-ganado.

\begin{tabular}{ccc}
\hline Cobertura & \multicolumn{2}{c}{$\%$} \\
\cline { 2 - 3 } & PC & Lignina \\
\hline Brachiaria dyctioneura & $6,70 \mathrm{~b}( \pm 0,45)$ & $4,78 \mathrm{~b}( \pm 0,35)$ \\
Centrosema macrocarpum & $11,15 \mathrm{a}( \pm 0,41)$ & $5,01 \mathrm{a}( \pm 0,57)$ \\
Sabana natural & $5,63 \mathrm{c}( \pm 0,77)$ & $4,40 \mathrm{~b}( \pm 0,28)$ \\
\hline
\end{tabular}

PC: proteína cruda. Letras diferentes en una misma columna señala diferencias estadísticamente significativas entre coberturas y sabana natural. En la tabla se expresa un valor promedio durante el ciclo de pastoreo y su desviación standard. (Duncan $\mathrm{p}<0.05)$.

En ese sentido, el contenido de PC ha sido utilizado como uno de los parámetros para medir la calidad de coberturas tropicales al estar correlacionado consistentemente con medidas del contenido de energía disponible en los forrajes, tales como la digestibilidad de la materia seca y el contenido de fibra (WILKINS, 2000).

Al respecto, CHACÓN (2007) señala valores generales de PC en hojas secas $(9.7 \%)$, los cuales varían dependiendo del tipo de especie, época del año y momento de corte. Estos valores contrastan con los observados en la sabana natural, conformada principalmente por Trachypogon spicatus y Andropogon selloanus, donde se presentan los mayores déficit de PC $(<7 \%)$. Sin embargo, hay autores que señalan otras concentraciones de PC en gramíneas nativas de sabanas, de acuerdo al momento del rebrote. Así MATA et al. (1985) reportan tenores de 2.50 a $13.01 \%$, TEJOS (1996) señala valores de 2.7 a 3.9\% y BALDIZÁN et al. (2006) de 2.80 a $10.50 \%$.

Otro parámetro bromatológico de calidad es la lignina, la cual se relaciona directamente con la edad de la planta por formar parte de la pared celular (HAFEZ Y DYER, 1969). En este sentido, el detrimento de la calidad de un forraje ocurre con el envejecimiento de las hojas afectando su calidad, la cual viene dada por la degradabilidad de la pared celular. Si bien la calidad de un pasto no está determinada por la cantidad de lignina per se, sino por el aumento de ciertos compuestos 
fenólicos, precursores de la lignina, como los ácidos p-cumárico y ferúlico que se unen a la pared celular (IBRAHIM et al., 2001), se utiliza la lignina como un indicador de madurez, digestibilidad y calidad del forraje.

Al estudiar el contenido de lignina durante el pastoreo se observó que la leguminosa $\mathrm{Cm}$ presentó mayores valores $(5.01 \pm 0.57 \%)$ en comparación con la Bd $(4,78$ $\pm 0,35 \%)$. Esto se debe a que las leguminosas tienden a tener contenidos de pared celular bajos y contenidos de lignina altos, mientras que en las gramíneas sucede lo contrario reportándose altos valores de pared celular y bajos de lignina (SENE et al., 2012). En este sentido, VELÁSQUEZ et al. (2009) mencionan valores para la Bd que oscilan de 5 a $8 \%$ de lignina en agroecosistemas de sabanas. Asimismo VALENCIAGA et al. (2009) han conseguido concentraciones de lignina en gramíneas que oscilan entre 4,2 y $8,9 \%$ de acuerdo al momento del corte y el tipo de manejo.

Por otra parte, los valores de FDA determinados en el material vegetal verde fueron de $41,88( \pm 5,46) \%$ en $\mathrm{Bd}, 50,62( \pm 3,34) \%$ en $\mathrm{Cm}$ y $64,51( \pm 2,73) \%$ en SN respectivamente, mientras que los de FDN fueron los siguientes: $51,51( \pm 3,23) \%, 58,19( \pm 3,95) \%$ y 67,55 $( \pm 6,45) \%$. Al respecto, ARSYADI et al (2014) señala que la FDA, es el porcentaje de las plantas altamente digeribles, las cuales tienden a ser mayores en la gramíneas en contraste con la leguminosa, por lo que a menores valores de FDA hay una mayor digestibilidad de la cobertura, cumpliéndose esto en la vegetación nativa de $\mathrm{SN}$, no así para la $\mathrm{Bd}$, la cual mostró menores valores de lignina, FDA y FDN en comparación con la leguminosa.

Las entradas de $\mathrm{P}$ dentro del agroecosistema por medio de las excretas, también están relacionadas con la calidad bromatológica del forraje que el rebaño consume durante el pastoreo (MURGUEITIO et al., 2015), siendo la lignina, así como el contenido de fibra detergente ácido y fibra detergente neutro, no solo indicadores de consumo voluntario, sino también indicadores de digestibilidad (GAVIRIA-URIBE et al., 2015, RAY et al., 2016).

De igual forma, los contenidos de PC, como la lignina son indicadores importantes al hacer el seguimiento del ciclaje de $\mathrm{P}$ en el agroecosistema suelo.-planta-animal, puesto que el aporte y entradas de este elemento dependerá de lo que consume el animal-calidad nutricional y bromatológica de la cobertura- durante el pastoreo, así como lo excretado por parte del mismo.

Contenido de fósforo en el suelo, las coberturas y en las excretas del ganado durante el pastoreo. En la Figura 1, se muestran los contenidos de P disponible
( $\mathrm{mg} \mathrm{kg}^{-1}$ ) en el suelo, el P orgánico en la hoja verde de las coberturas (Brachiaria dyctioneura, Centrosema macrocarpum) y de la vegetación nativa de la sabana natural, así como la presencia de este elemento en las excretas del ganado que pastoreó cada uno de los tratamientos durante los 110 días que duró el ensayo.

La concentración de $\mathrm{P}$ al inicio del ciclo de pastoreo en sus diferentes formas, tanto para el suelo $-P$ disponible-, como para la biomasa - $P$ orgánico- y las excretas - $\mathrm{P}$ total-, mostró diferencias estadísticamente significativas $(p<0.05)$, dependiendo del tipo de cobertura, posiblemente debido al efecto residual de la fertilización empleada inicialmente para establecer el ensayo. A su vez se observó una menor concentración de este elemento para los tres componentes en la sabana de vegetación natural (SN), excepto en el caso de las excretas, donde los contenidos de $\mathrm{P}$ total fueron similares a los mostrados por los animales que pastorearon en la $\mathrm{Cm}$. Al comparar entre coberturas hay una mayor disponibilidad de $\mathrm{P}$ en los suelos bajo el tratamiento con la gramínea Bd.
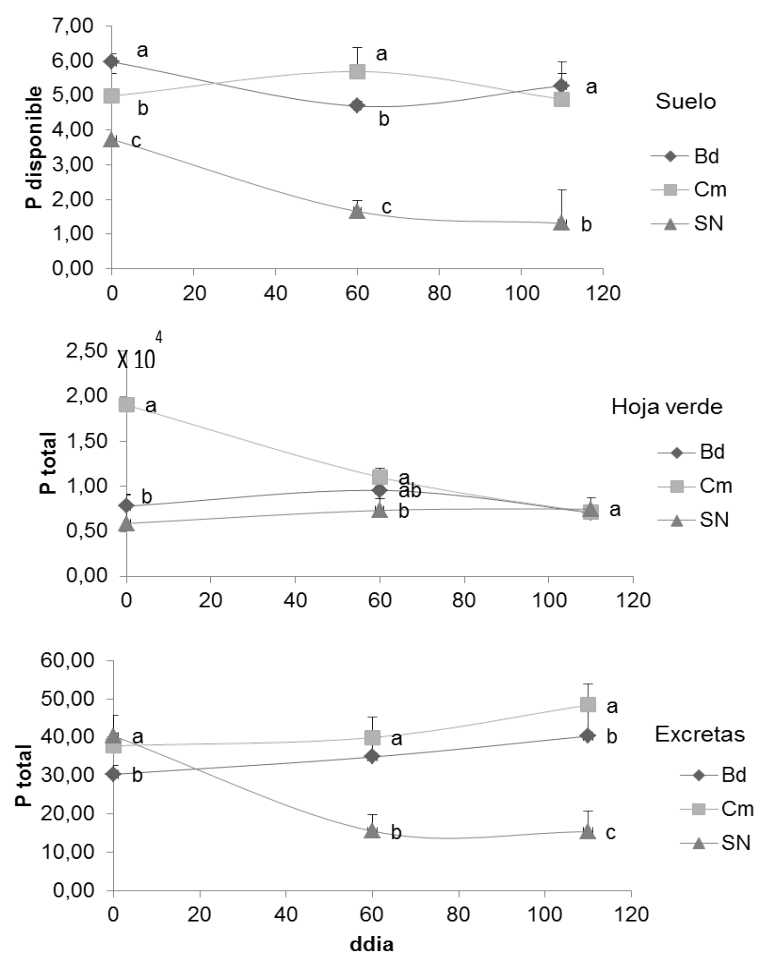

Figura 1.Dinámicas de $\mathrm{P}$ en el agroecosistema maíz-ganado asociado a la Brachiaria dyctioneura (Bd), Centrosema macrocarpum $(\mathrm{Cm})$ y sabana de vegetación natural (SN). Letras diferentes en cada momento de muestreo señalan diferencias estadísticamente significativas entre coberturas. Barras indican desviación estándar. Valores de $\mathrm{P}$ expresados en $\mathrm{mg} \cdot \mathrm{kg}^{-1}$. ddia: días después de introducidos los animales al ensayo. (Duncan $p<0.05$ ). 
A medida que transcurrió el pastoreo las diferencias en los contenidos de $\mathrm{P}$ tanto en el suelo, como en las coberturas y en las excretas, siguen siendo significativas y diferentes, favoreciendo claramente a las coberturas introducidas versus la vegetación nativa de sabana (SN). De igual forma, a los 60 días después de introducidos los animales (ddia), la disponibilidad del $P$ en el suelo es mayor en el tratamiento con $\mathrm{Cm}$. Al finalizar el ciclo de pastoreo se hace notar la diferencia en la disponibilidad de éste elemento favorecido por las pasturas introducidas, frente a la vegetación de SN.

Al respecto SING et al. (2010) señalan el efecto benéfico de gramíneas sobre la disponibilidad de $\mathrm{P}$ en suelos, debido a que conserva la humedad por formarse un colchón vegetal, disminuye el impacto del pisoteo del ganado y mejora la aireación de los suelos (BELTRAN et al., 2013). Por el hecho de tener mejor calidad nutricional en su biomasa, la Bd, si es bien manejada, considerando los períodos de recuperación y/o rotación que se establezcan al momento de ser sometida a pastoreo (DÍAZ, 2013), puede aumentar la producción y calidad de su biomasa, la cual puede ser aprovechada no solo por el mismo agroecosistema, al momento de descomponerse e incorporar el material vegetal de mejor calidad al suelo (SING et al., 2010), sino también en la alimentación del ganado, mejorando sus índices de productividad (ARGEL, 2006). De igual forma cabe destacar que al ser un suelo de $\mathrm{pH}$ ácido $(<5)$, la disponibilidad de éste elemento se ve comprometida por presentarse en formas no asimilables por la planta, sin embargo trabajos como LOZANO et al., (2010), indica mejoras e incrementos del pH por la introducción de coberturas, mejorando así las disponibilidad de este elemento en el suelo.

De igual forma, PETERS (2014) señala que de acuerdo al manejo de los suelos se puede acelerar los procesos de descomposición biótica y abiótica de la materia orgánica, facilitando que el $\mathrm{P}$ orgánico pase a formas disponibles para la planta (LOZANO et al., 2010, NOVAL-ARTILES et al., 2014) lo que posiblemente explica el hecho de que, a pesar del pastoreo intensivo y la elevada carga animal, el forraje aún presenta en la mayor parte de su dinámica, altos tenores en los contenidos de $\mathrm{P}$ en hoja, sin embargo al finalizar el ciclo de pastoreo las concentraciones de este elemento a nivel foliar no mostraron diferencias significativas entre las pasturas introducidas y la vegetación nativa de SN.

Al inicio del ciclo de pastoreo, las concentraciones de $\mathrm{P}$ total presente en las excretas, fueron análogas a las mostradas a nivel foliar para $\mathrm{Cm}$, asimismo se encontró, concentraciones de éste elemento, similares en las excretas del ganado para el caso de la pastura nativa de $\mathrm{SN}$. A medida que transcurrió el pastoreo los contenidos de $P$ en las excretas variaron, siendo mayores en los tratamientos bajo pastos mejorados, y manteniendo este mismo patrón hasta finalizar el ciclo de pastoreo. El monitoreo del $\mathrm{P}$ total en las excretas puede indicar que la incorporación de este elemento al ciclaje en el suelo es mayor cuando se introducen pasturas de mejor calidad nutricional, en contraste con un sistema bajo pasturas naturales, tal y como es señalado por MURGUEITIO et al. (2015), en agroecosistemas bajo pastoreo.

En el agroecosistema estudiado se debe mencionar que al momento de introducir el ganado, las coberturas se encontraban asociadas con el maíz y venían de un proceso de fertilización, lo que podría explicar las diferencias en términos de concentración de $\mathrm{P}$ entre las coberturas y oferta de este elemento cuando se inicia el pastoreo, otro de los aspectos importantes que hay que considerar es que las leguminosas suelen tener mayores contenidos de $P$ en sus tejidos (FRIESEN et al., 2013), por lo que el aporte de este elemento en el agroecosistema de la leguminosa, posiblemente sea mayor a diferencia de la $\mathrm{Bd}$ y la vegetación nativa de $\mathrm{SN}$.

De igual forma es importante destacar que la presión de pastoreo puede influir en los contenidos de $\mathrm{P}$ total extraíble y P orgánico del suelo (LÓPEZ-MÁRSICO et al., 2016). Se ha observado que cuando aumenta el nivel de fosfatos -fertilización, presencia de excretas o mineralización de la materia orgánica- se pueden producir minerales insolubles que afectan y disminuyen las formas inorgánicas disponibles (ZANOLETTI, 2016). Cabe destacar que, los cambios en las diferentes formas de $\mathrm{P}$ en el suelo, su balance y dinámica en el tiempo, están influenciados por factores como precipitación, exportación de la cosecha, pastoreo y tipo de vegetación la cual a su vez se relaciona directamente con la digestibilidad de la misma, y lo excretado por el ganado, lo cual pasa a formar parte del suelo (SINCLAIR et al., 1992).

Las dinámicas del $\mathrm{P}$ en los componentes suelo-plantaexcretas, muestran tener una interrelación, la cual viene dada por la disponibilidad de este elemento en el suelo y a su vez, por la concentración presente en las coberturas que son consumidas por parte del animal durante su alimentación. Tal es el caso de la gramínea donde al inicio del pastoreo, las concentraciones de $\mathrm{P}$ disponible en el suelo tienden a ser mayores en comparación con los suelos de $\mathrm{Cm}$ y vegetación nativa de $\mathrm{SN}$. Estas menores concentraciones de $\mathrm{P}$ disponible en los otros tratamientos puede deberse a la falta de humedad, propia de la época seca, donde si bien puede haber un efecto de mayor disponibilidad de este elemento por efecto de la cobertura, la planta limita su toma del suelo, por lo que las concentraciones de este elemento a nivel foliar tienden a ser menores, incidiendo directamente en el aporte, que este elemento puede hacer al momento de su defoliación. 
De manera general, el ganado que pastoreó en $\mathrm{Bd}$ y $\mathrm{Cm}$, muestra mayores contenidos de $\mathrm{P}$ total en las excretas, coincidiendo así también con las disponibilidades de este elemento en el suelo y su concentración en las hojas de las coberturas.

En la Tabla 2 se indican las correlaciones presentadas en la $B d, C m$ y vegetación nativa de $\mathrm{SN}$, respectivamente, para los contenidos de $\mathrm{P}$ en los componentes sueloplanta y excretas, así como variables bromatológicas y la incidencia en la respuesta animal en términos de ganancia diaria de peso (GDP).

En el tratamiento bajo $B d$, el $\mathrm{P}$ total presente en las excretas mostró correlaciones significativas $(p<0.05)$ con la concentración de $\mathrm{P}$ a nivel foliar $(r=0.65)$ y el $P$ disponible en el suelo $(r=0.64)$, sin embargo no presentó correlaciones significativas con otros parámetros medidos en el agroecosistema como FDA, FDN, lignina y GDP. EI P a nivel foliar tuvo una correlación positiva con la GDP. Mientras que la GDP se correlacionó negativa y significativamente $(p<0.01)$ con los contenidos de lignina en la hoja verde de la gramínea, indicando que a mayor contenido de lignina en la hoja, el peso del animal durante el pastoreo es menor.

La alta correlación en el tratamiento con la gramínea entre el $\mathrm{P}$ total presente en las excretas y la concentración de $\mathrm{P}$ en la biomasa de la cobertura, está relacionado con el hecho de que la actividad biológica que se genera durante la etapa inicial de la degradación aeróbica de la excreta de bovinos, influye sobre la dinámica del
$\mathrm{P}$ disponible en el suelo, debido a la variación de las condiciones fisicoquímicas del medio, favoreciendo los procesos de inmovilización-mineralización de éste elemento, resultados similares fueron encontrados por HERNÁNDEZ-HERNÁNDEZ et al. (2000), al comprobar una mayor disponibilidad de $P$ en el suelo, y a su vez una alta correlación con el tipo de cobertura utilizada así como con la presencia de bacterias solubilizadoras de P.

En la Tabla 3 se muestran las correlaciones entre la concentración de $\mathrm{P}$ en la hoja de $\mathrm{Cm}$, la disponibilidad de este elemento en el suelo y la GDP durante el pastoreo. Se observan más correlaciones significativas entre el $\mathrm{P}$ de las variables evaluadas en la leguminosa, en comparación con la gramínea. EI $\mathrm{P}$ total presente en las excretas bajo el tratamiento con $\mathrm{Cm}$, mostró correlaciones significativas con la concentración de $P$ en las hojas de la cobertura, el $P$ disponible en el suelo y a su vez la GDP, mientras que el $P$ de la biomasa indica una correlación mayor y significativa con la GDP, mientras que los contenidos de lignina están correlacionados altamente con FDA y FDN y negativamente con la GDP.

Si bien la $\mathrm{Cm}$, mostró de acuerdo a la literatura mayores contenidos de lignina en contraste con la $B d$ (CASTILLO et al., 2008, ARSYADI et al 2014), los resultados indican que hay una mejor incorporación del $\mathrm{P}$ por medio de las excretas en el suelo bajo ésta cobertura (LEÓNNÁJERA et al., 2014), lo que a su vez incide en una mayor disponibilidad de este elemento para ser tomado por la leguminosa.

Tabla 2. Correlaciones entre los contenidos de $\mathrm{P}$ de la cobertura Brachiaria dyctioneura, el suelo y la respuesta animal durante el pastoreo.

\begin{tabular}{ccccccc}
\hline Variables & P excretas & P biomasa & P suelo & Lignina & FDA & \\
\hline P excretas & & & & & \\
P biomasa & $0,65^{\star}$ & & & & \\
P suelo & $0,64^{\star}$ & $-0,15$ & 0,14 & & \\
Lignina & 0,01 & $-0,01$ & 0,13 & 0,30 & $0,76^{\star *}$ \\
FDA & 0,01 & 0,03 & 0,17 & 0,22 & $-0,75^{*}$ \\
FDN & 0,03 & 0,14 & $0,60^{*}$ & $-0,96^{\star *}$ & 0,23 \\
GDP & $-0,37$ & $0,60^{\star}$ & & \\
\hline
\end{tabular}

La significación se muestra con * significativo (Pearson. $p<0.05)$ y* altamente significativo $(p<0.01)$.

Tabla 3. Correlaciones entre los contenidos de $\mathrm{P}$ de la cobertura Centrosema macrocarpum, el suelo y la respuesta animal durante el pastoreo.

\begin{tabular}{|c|c|c|c|c|c|c|c|}
\hline Variables & P excretas & P biomasa & $\begin{array}{c}P \\
\text { suelo }\end{array}$ & Lignina & FDA & FDN & GDP \\
\hline \multicolumn{8}{|l|}{ P excretas } \\
\hline $\mathrm{P}$ biomasa & $0,52^{*}$ & & & & & & \\
\hline P suelo & $0,52^{*}$ & 0,48 & & & & & \\
\hline Lignina & $0,57^{*}$ & 0,44 & $-0,12$ & & & & \\
\hline FDA & 0,30 & $-0,04$ & $-0,03$ & $0,94^{\star *}$ & & & \\
\hline FDN & 0,25 & 0,31 & $-0,03$ & $0,96^{* *}$ & $0,98^{\star *}$ & & \\
\hline GDP & $0,77^{\star *}$ & $0,71^{\text {** }}$ & 0,06 & $-0,87^{\star *}$ & $-0,52^{*}$ & $-0,14$ & \\
\hline
\end{tabular}

La significación se muestra con * significativo (Pearson. $p<0.05)$ y* altamente significativo $(p<0.01)$. 
Estudios previos realizados por ALGUACIL et al. (2014) en este agroecosistema, indican la presencia de micorrizas asociadas a la leguminosa. Esto explica el hecho, de que junto a ser fijadora de nitrógeno, facilita la disponibilidad y toma del $\mathrm{P}$ desde el suelo hasta la planta (MARTíN y RIVERA, 2015).

En el agroecosistema las mayores concentraciones de $\mathrm{P}$ a nivel de biomasa se encontraron en la $\mathrm{Cm}$, seguido de la Bd y por último en la vegetación nativa de SN (Figura 1). Resultados similares se publicaron por HERNÁNDEZHERNÁNDEZ et al. (2000) quienes demostraron la presencia de organismos solubilizadores de $\mathrm{P}$, lo cual sugiere un efecto positivo sobre la toma de $\mathrm{P}$ por parte de las plantas en estos suelos, que junto a la fijación simbiótica de $\mathrm{N}$ del aire por bacterias simbióticas, ofrece grandes perspectivas de suministrar $\mathrm{P}$ a la planta por lo que actúan en sinergia a fin de suplir al agroecosistema, de un mecanismo biológico importante en el ciclaje de este elemento.

En la Tabla 4, bajo vegetación nativa de SN, el resultado de las correlaciones fue diferente al contrastarlo con las coberturas introducidas. Tal es el caso del P en la biomasa, el cual mostró correlaciones altas y significativas $(p<0.01)(r=0.88)$ con la lignina, lo que podría indicar que en el momento evaluado, por ser época seca las mayores concentraciones de $\mathrm{P}$ presente, estaban relacionados con los mayores contenidos de lignina, y a su vez su biomasa en gran parte es seca lo que incide negativa y significativamente en la GDP durante el pastoreo.

En la sabana natural no se evidencian correlaciones del $P$ que potencialmente se incorporará por las excretas con el $\mathrm{P}$ disponible del suelo ni con el de la biomasa verde; sin embargo, es clara la relación directa, entre la mayor correlación en disponibilidad del $\mathrm{P}$ en el suelo durante la época de pastoreo, así como las correlaciones negativas y significativas de este elemento en la planta, lo que muestra que a medida que la planta toma el $\mathrm{P}$ disponible del suelo, éste elemento tiende a disminuir su presencia en el mismo (GONZÁLEZ et al., 2016).

La dinámica del $P$ en las relaciones suelo-planta, se facilitan por diversos factores, entre ellos la humedad del suelo, variable que es favorecida sobre todo en las pasturas introducidas ya que se hace un colchón de biomasa en el potrero reteniendo parte de la humedad en el suelo, a diferencia de lo que puede ocurrir en la sabana de vegetación nativa (FRIESEN et al., 2013).

Otro de los aspectos importantes a destacar es el hecho, que las pasturas introducidas presentan mayor calidad nutricional, siendo empleado para ello, algunas variables bromatológicas como proteína cruda y lignina, las cuales son un indicativo de la calidad y aceptabilidad de la cobertura utilizada como forraje, durante el pastoreo, por lo que el aporte vía descomposición en el agroecosistema será mayor al contrastarlo con la vegetación de SN, donde el aporte de este elemento está limitado por la calidad y lignificación de la biomasa forrajera (ABREU, 2011; TALAVERA et al., 2016).

\section{Conclusiones}

La concentración del $\mathrm{P}$ en el agroecosistema varía de acuerdo al tipo de cobertura, siendo potencialmente mayor el ciclaje de este elemento, en la Brachiaria dyctioneura y la Centrosema macrocarpum. La concentración de $\mathrm{P}$ en las excretas está relacionado directamente con el $P$ presente a nivel foliar y en el caso de la leguminosa y la sabana natural con la ganancia diaria de peso, mientras que la Brachiaria dyctioneura, indica una correlación con el $P$ presente en el suelo. En éste tipo de agroecosistemas en época seca, la disponibilidad de los nutrientes en el suelo se reduce y en consecuencia el rendimiento de los pastos también, ocasionando pérdidas de peso en los animales, y afectado el proceso productivo del agroecosistema, por lo que es imperativo continuar con el estudio de estrategias que permitan mejorar la disponibilidad de éste elemento que es limitante en suelos de sabana y que afecta significativamente las relaciones suelo-planta-animal.

Agradecimientos: Universidad Nacional Experimental Simón Rodríguez a través de proyecto: "Manejo Agroecológico de suelos de sabanas bien drenadas con unidades de producción cereal-ganado",G-2002000398, el cual fue desarrollado desde el Instituto de Estudios Científicos y Tecnológicos (IDECYT) y financiado por el Fondo Nacional de Ciencia Tecnología e Industrias Intermedias (FONACIT).

Tabla 4. Correlaciones entre los contenidos de P de la cobertura de la vegetación nativa de SN, el suelo y la respuesta animal durante el pastoreo.

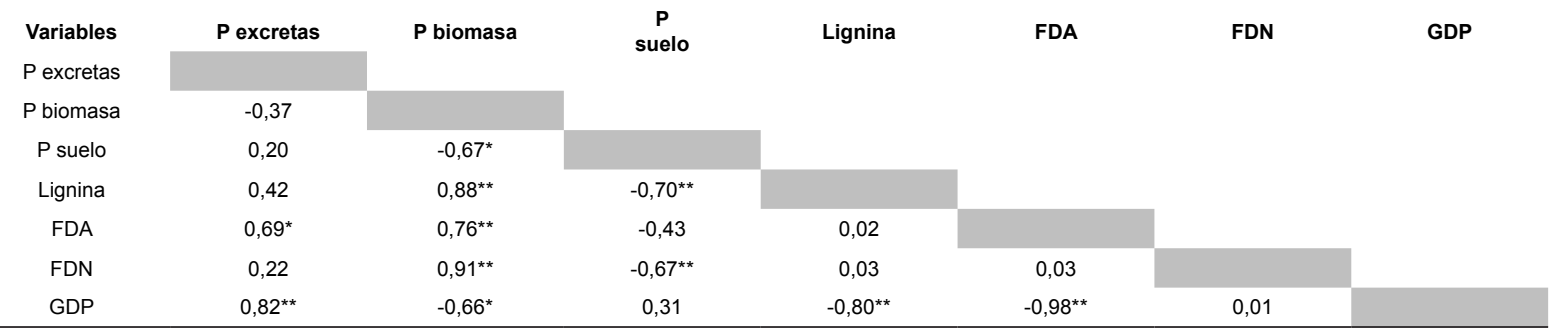

La significación se muestra con * significativo (Pearson. $p<0.05) y$ ** altamente significativo $(p<0.01)$. 


\section{Referencias}

ABREU, X. 2011. Evaluación de cuatro leguminosas como barbecho mejorado durante dos épocas del año para ser usadas como cultivos de cobertura. Venesuelos 4 (1 y 2):8-13.

ALGUACIL, M.d.M.; TORRECILLAS, E.; LOZANO, Z.; TORRES, M.P.; ROLDÁN, A. 2014. Prunus persica crop management differentially promotes arbuscular mycorrhizal fungi diversity in a tropical agro-ecosystem. PloS one 9 (2):e88454.

ARGEL, P.J. 2006. Contribución de los forrajes mejorados a la productividad ganadera en sistemas de doble propósito. Arch. Latinoam. Prod. Anim, 14 (2): 65-72.

ARSYADI, A.; LUKI, A.; PDMH, K.; MA, C.; DA, A. 2014. Evaluation, Productivity and Competition of Brachiaria decumbens, Centrosema pubescens and Clitoria ternatea as Sole and Mixed Cropping Pattern in Peatland's. Indonesian Journal of Animal and Veterinary Sciences, 19(2):p-81.

BALDIZÁN A., DOMINGUEZ C., GARCÍA D., CHACÓN E., AGUILAR L. 2006. Metabolitos secundarios y patrón de selección de dieta en el bosque deciduo tropical de los llanos centrales venezolanos. Zootecnia Tropical 24 (3):213-232.

BELTRAN, M.J.; BRUTTI, L.; ROMANIUK, R.; BACIGALUPPO, S.; BORRERO, G.; FRIESEN, D.; OBERSON, A.; RODRÍGUEZ, M.; RAO, I.M. 2013. Destinos del Fósforo Aplicado como Fertilizante en Oxisoles de los Llanos Orientales de Colombia: Fraccionamiento Secuencial. Sistemas Agropastoriles 159.

BROWN, S. 1997. Estimating biomass and biomass change of tropical forests: a primer. Vol 134. Food \& Agriculture Org. Illinois, USA.

CASTILLO A.; MORENO G.; GARAY E. 2008. Producción de forraje en los pastos Brachiaria decumbens cv. Amargo y Brachiaria brizantha cv. Toledo, sometido a tres frecuencias y a dos intensidades de defoliación en condiciones de Piedemonte Llanero Colombiano. Rev.Fac.Nal.Agr.Medellín 6 (1):4336-4346.

CASTELLARO, G.; ULLRICH, T.; WACKWITZ, B.; RAGGI, A. 2007. Composición botánica de la dieta de alpacas (Lama pacos L.) y llamas (Lama glama L.) en dos estaciones del año, en praderas altiplánicas de un sector de la provincia de Parinacota, Chile. Agric. Téc. (Chile) 64:353-364.

CHACÓN, E. 2007. Programas de desempeño tecnológico en recursos alimentarios para la producción con rumiantes a pastoreo. I simposio para tecnologías para la ganadería de los llanos de Venezuela. Capítulo IV 251-284.

DI RIENZO J.A.; CASANOVES F.; BALZARINI M.G.; GONZALEZ L.; TABLADA M. y ROBLEDO C.W. InfoStat versión 2011. Grupo InfoStat, FCA, Universidad Nacional de Córdoba, Argentina. URL http://www.infostat.com.ar

DÍAZ, E.C.G. 2013. Modalidades de producción, manejo animal y de pasturas en condiciones de sabanas bien drenadas de Venezuela. Págs. 117-124. En: Manejo de Pastos y Forrajes Tropicales. Editorial Fundación Girarz. Caracas,Venezuela.

DUNCAN, A 1974. Quality Control and Industrial Statistics. $4^{\text {th }}$ Ed. Irwin Homewoods III. Illinois, USA.

EWEL, J; MADRIZ, A.; TOSI, J. 1976. Zonas de vida de Venezuela. Memoria explicativa sobre el mapa ecológico $2^{\mathrm{a}}$ edición. MAC-FONAIAP, Caracas.

FRIESEN, D.K.; RAO, I.M.; THOMAS, R.J.; OBERSON, A.; SANZ, J.I. 2013. Adquisición y reciclaje de fósforo en sistemas de cultivos y pasturas en suelos tropicales de baja fertilidad. Sistemas Agropastoriles 1:147-158.

GAVIRIA-URIBE, X.; NARANJO-RAMÍREZ, J.F.; BOLÍVAR-VERGARA, D.M.; BARAHONA-ROSALES, R. 2015. Consumo y digestibilidad en novillos cebuínos en un sistema silvopastoril intensivo. Archivos de zootecnia 64 (245):21-27.

GONZÁLEZ, A.; SZOSTAK, J.; MOREL, J.; ISHIWATA, T. 2016. Efecto de rotaciones de cultivos en la evolución del contenido de cationes y el pH de suelos arcillosos bajo siembra directa, en el Distrito de Capitán Miranda-Paraguay. Tecnología Agraria 1 (1):51-56. 
HERNÁNDEZ-HERNÁNDEZ, RM; FLORENTINO A.; LÓPEZ-HERNÁNDEZ D. 2000. Efecto de la siembra directa y la labranza convencional en la estabilidad estructural y otras propiedades físicas de un suelo Ultisol en el estado GuáricoVenezuela. Agronomía Tropical. 50 (1):9-29.

IBRAHIM, M; FRANCO M.; PEZO D.; CAMERO A.;ARAYA, J. 2001. Promoting intake of Cratylia argentea as a dry season supplement for cattle grazing Hyparrhenia rufa in the subhumid tropics. Agroforestry Systems 51:167-175.

LASCANO C.; PÉREZ R.; PLAZAS C.; MEDRANO J.; PEREZ O.; ARGEL J. 2002. Pasto Toledo (Brachiaria brizantha): Gramínea de crecimiento vigoroso para intensificar la ganadería colombiana. CIAT. Bogotá-

LEÓN-NÁJERA, J.A.; GÓMEZ-ÁLVAREZ, R.; HERNÁNDEZ-DAUMÁS, S.; ÁLVAREZ-SOLÍS, J.D.; PALMA-LÓPEZ, D. J. 2014. Mineralización en suelos con incorporación de residuos orgánicos en los altos de Chiapas, México. Ecosistemas y Recursos Agropecuarios, 22(2):10-19.

LÓPEZ-MÁRSICO, L.; OYARZABAL, M.; ALTESOR, A.; PARUELO, J.M. 2016. Efecto del pastoreo sobre la productividad primaria neta subterránea de un pastizal templado. Ecología Austral 26 (3):256-263.

LOZANO Z.; MOGOLLÓNA.; HERNÁNDEZR.M.; BRAVO C.; OJEDAA.; TORRESA.; RIVERO C.; TORO M. 2010. Cambio en las propiedades químicas de un suelo de sabana por la introducción de pasturas mejoradas. Bioagro 22: $135-144$.

MACHADO, W. 2000. Planificación y análisis de experimentos de campo en grandes parcelas sin repetición. Universidad Central de Venezuela. Facultad de Agronomía. Revista de la Facultad de Agronomía. Alcance (59):73.

MARTíN, G.M.; RIVERA, R. 2015. Influencia de la inoculación micorrízica en los abonos verdes. Efecto sobre el cultivo principal. Estudio de caso: el maíz. Cultivos Tropicales 36:34-50.

MATA, D.; HERRERA, P.; BIRBE, B. 1996. Low inputs livestock systems for the Trachypogon sp. Ecotropicos 9 (2):83-100.

MATA D.; MORENO E.; DE ROJAS N. 1985. Efecto de la edad sobre la composición química del Trachypogon spp en una sabana del sureste del estado Guárico. Zootecnia Tropical 3 (12):29-48.

MURPHY J.; RILEY H.P. 1962. A modified single solution method for the determination of phosphate in natural waters. Analitical. Chemistry Acta 27:31-36.

MURGUEITIO, E.; FLORES, M.X.; CALLE, Z.; BARAHONA, J.D.C.R.; MOLINA, C.H.; URIBE, F. 2015. Intensivos en América Latina. Sistemas Agroforestales 59.

NOVAL-ARTILES, E.; GARCÍA-DÍAZ, J.R.; GARCÍA-LÓPEZ, R.; QUIÑONES-RAMOS, R.; MOLLINEDA-TUJILLO, Á. 2014. Caracterización de algunos componentes químicos, en suelos de diferentes agroecosistemas ganaderos. Centro Agrícola 41 (1):25-31.

PETERS, W. 2014. Suelos y ecosistemas del trópico húmedo. Revista de la Facultad de Agronomía 2 (3):69-85.

PORTELA, S.I.; RESTOVICH, S.B.; GONZALEZ, H.M.; TORTI, M. J. 2016. Reducción del drenaje profundo y la lixiviación de nitrógeno en rotaciones agrícolas con cultivos de cobertura. Ecología Austral 26 (3):212-220.

RAMIA, M. 1967. Tipos de Sabanas en los llanos de Venezuela. Bol. Soc.Ven. Cien. Nat. 27:264-288.60-272.

RAMÍREZ-IGLESIAS E. 2015. Sincronización de los componentes suelo-planta-animal en agroecosistemas de sabanas bien drenadas del estado Guárico. Tesis doctoral. Instituto de Zoología Tropical, Facultad de Ciencias. Universidad Central de Venezuela. Venezuela.

RAY, J.R.; BENITEZ, D.B.; GARCIA, F.G.; VEGA, A.V. 2016. Consumo voluntario, digestibilidad y balance de $\mathrm{N}$ y nutrientes de vacas criollas en pastoreo racional en el Valle del Cauto Cuba. Revista Amazónica Ciencia y Tecnología 5 (2):146-158.

RIERA, J.; GUERRERO, I. 1984. Caracterización agroecológica de la región oriental de Guárico. Sub estación Experimental Valle de la Pascua. FONAIAP. (Inedito). 
RODAS-GONZÁLEZ, A.; VERGARA-LÓPEZ, J.; ARENAS DE MORENO, L.; HUERTA-LEIDENZ, N.; PIRELA, M.F. 2006. Características al sacrificio, rasgos de la canal y rendimiento carnicero de novillos Criollo Limonero sometidos a suplementación durante la fase de ceba a pastoreo. Revista Científica 16 (4):315-324.

SANABRÍA, D.; ACUÑA-SILVA R.; MARCANO M.; BARRIOS R.; RIVAS, E.; RODRÍGUEZ I. 2006. Evaluación de tres sistemas de labranza en la recuperación de una pastura degradada de Brachiaria humidicola. Zootecnia Tropical 24 (4):417-433.

SEMMARTIN, M.; DIBELLA C.; GARCÍA S. 2010. Grazing-induced changes in plant species composition affect plant and soil properties of grassland mesocosmd. Plant Soil 328:471-481.

SENE G.; SAMBA-MBAYE R.; THIAO M.; KHASA D.; KANE A.; MANGAA.; MBAYE M.; SYLLA S. 2012. The abundance and diversity of legume-nudulating rhizobia and arbuscular mycorrhizal fungal communities in soil from deforest and manmade forest systems in a semiarid Sahel region in Senegal. European Journal of Soil Biology 52: 30-40.

SINCLAIR, R.; WEBBE L.; ROMEROA. 1992. Potencial del rastrojo de maíz con leguminosa en la alimentación del ganado de engorde. Agronomía mesoamericana 3:45-57.

SING S.; KUNDU S.; MAITY S. 2010. Relative intake, eating pattern, nutrient digestibility, nitrogen metabolism, fermentation pattern and growth performance of lambs fed organically and inorganically produced cowpea hay-barley grain diets. Tropical grassland 44:55-61.

SOEST, VAN P. 1994. Nutritional ecology of the ruminant. CornellUniversity. 2 ed. USA.

STEYERMARK, A.; HORWITZ, W. 1977. Official methods of analysis of the Association of Official Analytical Chemists. 94-94 p.

TALAVERA, Á.N.; SÁNCHEZ, N.R.; MEMBREÑO, J.J.; AGUILAR, C.D.; ARAICA, B.M. 2016. Calidad de plántulas de tres especies forrajeras (Moringa oleifera Lam., Leucaena leucocephala y Cajanus cajan) en condiciones de vivero. La Calera 14(22):12-21.

TEJOS, R. 1996. Carbohidratos no estructurales en dos gramíneas nativas de sabanas bien drenadas. Archivos Latinoamericanos de Producción Animal 4 (2):125-134.

THOMAS, R.J.; ASAKAWA, M.M. 1993. Descomposition of leaf litter from tropical forage grasses and legumes. Soil Biol. Biochem. 25:1351.

VALENCIAGA D.; HERRERA R.; OLIVEIRA S.; CHONGO B.; TORRES V. 2009. Composición monomérica de la lignina de Penisetum purpureum vc. Cuba CT-115 y su variación con la edad del rebrote. Revista Cubana de Ciencias Agrícolas 43 (3):315-319.

VELÁSQUEZ, R.; PEZO, D.; SKARPE, C.; IBRAHIM, M.; MORA-DELGADO, J.; BENJAMIN, T. 2009.Selectividad animal de forrajes herbáceos y leñosos en pasturas seminaturales en Muy Muy, Nicaragua. Agroforestería en las Américas 47:51-60.

WATABLE, F.; OLSEN S. 1965. Test o fon acid arcorbic methods for determining phosporus in water and NaHCO3 extracts from soil. Science society of American proceeding (29):677-678.

WILKINS R. 2000. Forages and Their Role in Animal Systems. In:Givens, D.; Owen E.; Axford R.; y Omed H. (eds) Forage Evaluation in Rumiant Nutrition. New York and Oxford 1-12.

ZANOLETTI, L. E. 2016. Evaluación de las propiedades químicas, físicas y biológicas del suelo destinado a la ganadería vacuna, con cultivo de Pennisetum purpureum cv Cuba CT-115 (Doctoral dissertation, Universidad Central" Marta Abreu" de Las Villas. Facultad de Ciencias Agropecuarias. Departamento de Agronomía. 